\title{
Transport due to Transient Progressive Waves
}

\author{
JUAN M. RESTREPO \\ Department of Mathematics and College of Earth, Ocean and Atmospheric Sciences, Oregon State University, \\ Corvallis, Oregon, and Kavli Institute of Theoretical Physics, University of California, Santa Barbara, Santa \\ Barbara, California \\ JORGE M. RAMIREZ \\ Departamento de Matemáticas, Universidad Nacional de Colombia Sede Medellín, Medellín, Colombia
}

(Manuscript received 8 April 2019, in final form 7 July 2019)

\begin{abstract}
Making use of a Lagrangian description, we interpret the kinematics and analyze the mean transport due to numerically generated transient progressive waves, including breaking waves. The waves are packets and are generated with a boundary-forced, air-water, two-phase Navier-Stokes solver. These transient waves produce transient transport, which can sometimes be larger than what would be estimated using estimates developed for translationally invariant progressive waves. We identify the critical assumption that makes our standard notion of the steady Stokes drift inapplicable to the data and explain how and in what sense the transport due to transient waves can be larger than the steady counterpart. A comprehensive analysis of the data in the Lagrangian framework leads us to conclude that much of the transport can be understood using an irrotational approximation of the velocity, even though the simulations use Navier-Stokes fluid simulations with moderately high Reynolds numbers. Armed with this understanding, it is possible to formulate a simple Lagrangian model that captures the mean transport and variance of transport for a large range of wave amplitudes. For largeamplitude waves, the parcel paths in the neighborhood of the free surface exhibit increased dispersion and lingering transport due to the generation of vorticity. We examined the wave-breaking case. For this case, it is possible to characterize the transport very well, away from the wave boundary layer, and approximately using a simple model that captures the unresolved breaking dynamics via a stochastic parameterization.
\end{abstract}

\section{Introduction}

The mean transport due to progressive waves here refers to the mean Lagrangian velocity generated by the waves. Longuet-Higgins (1953) presented a derivation of the mean Lagrangian transport, due to monochromatic waves, as an asymptotic series of averaged parcel paths and related the terms in the Lagrangian series to a series in the Eulerian framework. The asymptotic parameter was identified as the small distance traveled by a fluid parcel over a period of the wave motion. The first nonzero term in the series of the mean Lagrangian velocity is referred to as the Stokes drift velocity. [Stokes (1847) had derived the lowest-order approximation of the mean transport due to monochromatic progressive waves some nearly a century earlier.] Reviews and

Corresponding author: Juan M. Restrepo, restrepo@ math.oregonstate.edu analysis of wave-generated residual flows are found in Taylor and van den Bremer (2016) and van den Bremer and Breivik (2017). An alternative derivation of the mean transport can be accomplished via the generalized Lagrangian mean approach (Andrews and McIntyre 1978), wherein the asymptotic series is developed based upon the more geometric displacement vector [see Bühler (2014) for a detailed description of this approach].

How mean transport due to waves affect the dynamics of oceans at time and space scales larger than waves has been the subject of considerable attention. LonguetHiggins and collaborators (cf. Longuet-Higgins and Stewart 1960, 1962, 1964; Longuet-Higgins 1970a,b) proposed a radiation stress to account for wave generated transport on spatiotemporal scales larger than the waves. They also examined specific ways in which the stress or the interaction of the stress with a background current plays out in a variety of different important geophysical flows. The interaction of the residual flow 
due to gravity waves and the mean flow that makes up what is known as Craik-Leibovich (CL) theory [see Leibovich (1983) for a review] provides an alternative vortex force formulation to the radiation stress for the coupling. The vortex force enters the momentum equation as a contribution to the cross product of the total fluid rotation with the transport velocity, as approximated by the mean Eulerian velocity plus the Stokes drift velocity. A Bernoulli term also enters the momentum equation as an adjustment to the pressure gradient. [Similarities and differences between the radiation stress formulation and the vortex force formulation are detailed in Lane et al. (2007).] The destabilizing effect of the waves on currents in the CL theory forms the conceptual basis for the generation of Langmuir circulation, later generalized in McWilliams et al. (1997) to describe what is now known as Langmuir turbulence (see Teixeira and Belcher 2010). The $\mathrm{CL}$ theory was extended to capture the wave-driven circulation, in McWilliams and Restrepo (1999) and forms the basis for a shallow-water conservative dynamic of waves and currents (McWilliams et al. 2004).

This work is concerned with the mean transport due to transient progressive waves of small as well as of large amplitude. To this end, we will be applying ensemble characterizations to describe the average transport.

Mean transport due to wave packets is derived in van den Bremer and Breivik (2017). The transient progressive waves we consider are focusing wave packets. The transient waves examined here are not amenable to the analysis presented in Taylor and van den Bremer (2016) concerning wave groups, because the data do not have the requisite analysis time scale separation. We will demonstrate that the estimate that leads to the classical Stokes drift formula, which relies critically on an assumption in the wave statistics, does not hold generally for the numerically generated transient waves under consideration. This is at the heart of the explanation of why transient waves may produce significantly more transport than progressive waves, albeit localized in space and time. To make this point we will contrast our analysis to the more familiar residual flow due to steady, translationally invariant progressive waves.

The analysis will focus on characterizing and interpreting the transport of a specific set of numerically generated experiments of progressive waves, including breaking waves. The numerically generated transport considered in this paper revisits the transport results reported in Deike et al. (2017, hereafter DPM17). We use the same data, in fact. The numerical data are solutions to the boundary-forced Navier-Stokes equations, approximated by numerical means, for a heavy fluid (water) and an overlying light fluid (air). The waves were generated by a transient forcing boundary condition and were absorbed by the opposing boundary condition. Hence, we do not encounter some of the difficulties that arise in the real setting with regard to defining a mean transport, where waves may not have clean starting or ending times. We will make use of a Lagrangian description of the fluid flow in order to characterize and understand the transport. The Lagrangian parcel paths are computed using interpolation from the grid Eulerian velocity. The focus in DPM17 is on the transport due to wave breaking progressive waves and in the phenomenon of wave riding, in particular. We will touch upon this topic but our emphasis will be on characterizing transport of small and large transient progressive waves, as a function of depth and of wave slope, a parameter that controls the wave amplitude generated by the time dependent boundary condition.

The numerical data and its generation appear in section 2. In section 3 transport due to progressive monochromatic waves is summarized. Doing so gives us the opportunity to focus on the key distinction between transient and steady progressive wave transport, namely, an assumption critical for approximating the transient transport in terms of the series expansion leading to the familiar progressive wave transport derived in LonguetHiggins (1953), as modified in Restrepo (2007) to include unresolved processes parameterized by diffusion processes. Section 4 describes how transient mean transport is computed from the numerical data and proceeds to describe the transient transport. We contrast our analysis from the one provided in DPM17, which is based upon the same data. The kinematics of the parcels suggests a two-parameter model for the parcel dynamics. The model is described in section 5 and when tuned, recovers qualitatively the salient features of the mean transport and the dispersion ${ }^{1}$ in the data anywhere in the tank and for most wave amplitudes. The model is based upon the parcel kinematics and thus incorporates the fundamental aspects of the parcel dynamics that contribute to the mean transport and its variance. A summary of results appears in section 6 .

\section{Generation of numerical progressive wave Lagrangian paths}

In DPM17 the authors employ Gerris (see Popinet 2009), a Navier-Stokes equation solver, to obtain

\footnotetext{
${ }^{1}$ The term dispersion is used to describe the frequency dependence of the speed of waves. We will denote this type of dispersion by wave dispersion, in order to distinguish it from our other usage, to connote statistical spreading or variability.
} 
approximations of the motion of an air/water fluid under the action of a downward gravity force with magnitude $g$. Time is denoted by $t \geq 0$, and the simulation runs until $t=T_{f}=35 \mathrm{~s}$. The computations are done in two space dimensions with transverse coordinate denoted by $x ; z$ is the vertical coordinate, which increases upward from the quiescent reference level, $z=0$. The "tank" extent is $24 \mathrm{~m}$, and the depth of the water-filled tank is $h=1 \mathrm{~m}$. The fluid is subjected to a time dependent "paddle" forcing boundary condition at $x=0$ generating a wave packet which dissipates at the other end of the tank by absorbing boundary conditions. Zero velocity boundary conditions are imposed at the bottom of the tank, $z=-h$, and at $z=h$, the top of the domain. The NavierStokes solver uses the freshwater and air kinematic viscosity ratio and the computations reach Reynolds numbers on the order of 40000 (further details of the numerical generation of the flow and the parcel paths are found in DPM17 and references contained therein). At rest, initial conditions are invoked in all of the numerical simulations. The Eulerian velocity is denoted by $\mathbf{q}(x, z, t)$, and the free surface is $z=\eta(x, t)$.

Throughout this study we will make reference to the following, which we denote as the "linearized wave solution":

$$
\begin{aligned}
\eta^{w}(x, t)= & \sum_{n=1}^{N} a_{n} \cos \left[k_{n}\left(x-x_{b}\right)-\omega_{n} t\right], \\
\mathbf{u}^{w}(x, z, t)= & \nabla \phi(x, z, t), \quad \text { where } \\
\phi(x, z, t)= & -\sum_{n=1}^{N} \frac{a_{n} \omega_{n}}{k_{n}} \frac{\cosh \left[k_{n}(z+h)\right]}{\sinh \left(k_{n} h\right)} \\
& \times \sin \left[k_{n}\left(x-x_{b}\right)-\omega_{n} t\right],
\end{aligned}
$$

where $x_{b}=12 \mathrm{~m}$ and $t_{b}=25 \mathrm{~s}$ are the "focusing" position and time, respectively. The velocity $\mathbf{u}^{w}$ is an Eulerian velocity, and $\phi$ and $\eta^{w}$ are the velocity potential and sea elevation that describe an irrotational, incompressible infinitesimal-amplitude progressive wave packet.

In the simulations, the paddle is driven by the vector field $\mathbf{u}^{w}(0, z, t)$ in (1) with $N=32$. The prescribed paddle forcing produces a wave packet which reaches its highest steepness at a prescribed focusing position $x_{b}$ and focusing time $t_{b}$ (see DPM17). The simulation used the wave dispersion relation for angular frequencies $\omega_{n}=\sqrt{g k_{n} \tanh \left(k_{n} h\right)}$, where the $k_{n}$ are the wavenumbers. The wavenumbers $k_{n}$ were found using the wave dispersion relation and the frequencies chosen as follows: The component frequencies $\nu_{n}=0.5458+n \delta_{\nu} \mathrm{Hz}$, where $\delta_{\nu}=0.0222 \mathrm{~Hz}$. The central frequency is denoted $\nu_{c}=\omega_{c} / 2 \pi=0.89 \mathrm{~Hz}$. The wave dispersion is enforced at the paddle, as are the wave component amplitudes.
The paddle amplitudes $a_{n}$ were prescribed as follows: DPM17 employ the slope $S$, as an ordering parameter in the simulations. Here $S=\sum_{n=1}^{N} k_{n} a_{n}=N s$, and $s=k_{n} a_{n}$ is constant for $n=0,1, \ldots, N-1$. In the specification of each run the slope $S$ is fixed. The nature of the paddle forcing is such that large $S$ corresponds to high wave slopes as well as to a more transient flow. The data include simulations for the following cases

$$
\begin{aligned}
& S \in\{0.16,0.192,0.256,0.288,0.32,0.336,0.352, \\
&0.368,0.384,0.4,0.416\}
\end{aligned}
$$

with breaking occurring for $S \geq S_{0}=0.336$.

Parcel paths $\mathbf{Z}(t)=[X(t), Z(t)]$ were computed by an explicit first-order-in-time tracer advection scheme. The Lagrangian velocity at time $t$ was approximated using second-order interpolation of the grid Eulerian velocity field computed via the Navier-Stokes (Gerris) solver

$$
\dot{\mathbf{Z}}(t) \approx \mathbf{q}[\mathbf{Z}(t), t] .
$$

Figure 1 shows the starting locations of all paths $\mathbf{Z}(t=0)$ considered in the analyses that follow. Throughout this work we will connote the numerically generated outcomes as data. The shading scheme organizes the parcels by their position at the starting time. Our analyses focus on paths with $Z(0)$ within $10 \mathrm{~cm}$ of the surface. The gray area nearest to the surface will be henceforth referred to as the "near surface" paths. For large $S$ some parcel paths within the near surface, exhibit complex dynamics such as ejection and wave riding. Ejection refers to the loss of continuity of the free surface and possible generation of water spray. Wave riding refers to parcels residing for an extended time on the free surface of the waves (see Pizzo 2017). For the diagnosis of transport leading to Fig. 2, all of the parcel paths with initial positions shown as points in Fig. 1 were taken into account. In formulating a model for transport and dispersion (see section 5), we focused on paths starting within the gray area in the middle, $\left|X(0)-x_{b}\right|<\lambda / 2$ where $\lambda=1.96 \mathrm{~m}$ is the wave wavelength.

\section{Transport due to breaking monochromatic progressive waves}

To contrast the transient case we first review transport dynamics under the action of steady monochromatic progressive waves. This review will also prove helpful in reinterpreting the results on transport due to wave breaking presented in DPM17.

The Lagrangian velocity will be denoted by $\mathbf{U}(t)=$ $\dot{\mathbf{Z}}=[U(t), W(t)]$. The Stokes drift velocity is the lowestorder estimate of the mean Lagrangian velocity due to 


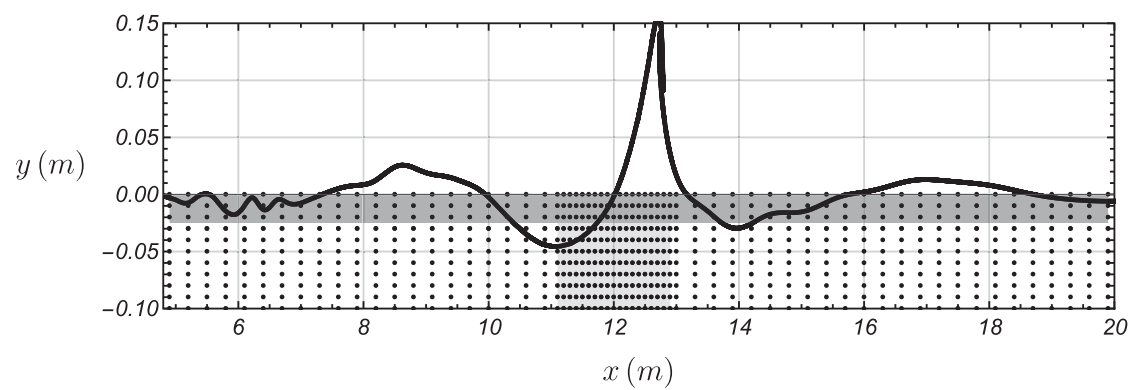

FIG. 1. Detail of the numerical tank, featuring the initial positions $\mathbf{Z}(0)$ of all of the parcel paths (dots) used in the analyses. The free surface of the wave right before breaking is shown, for the case $S=0.38$. The region with parcels with initial positions belonging to the nearsurface (approximately no deeper than $3 \mathrm{~cm}$ below the quiescent surface) is shaded in gray. Parcels starting in the upper gray zone may spend some portion of their history on the sea surface, or for some large values of $S$, be ejected during a breaking wave event. The zone that marks the boundary of parcel paths starting in the zone $\left|X(0)-x_{b}\right|<\lambda / 2$ is shown in lighter gray and has a higher density of parcel paths.

time periodic irrotational infinitesimal waves. For progressive waves of amplitude $a_{c}$, the Stokes drift is constant and proportional to $a_{c}^{2}$ (see Longuet-Higgins 1953). In Restrepo (2007) this result was extended to include an additive stochastic velocity component. When properly nondimensionalized, the Eulerian velocity is decomposed as

$$
\mathbf{q}(x, z, t)=\mathbf{q}^{D}(x, z, t)+\varepsilon \mathbf{u}^{w}(x, z, t)+\varepsilon^{2} \mathbf{q}^{C}(x, z, t, X, T),
$$

where $\mathbf{q}^{D}$ represent processes that are not resolved at the gravity wave scale, including contributions due to wave breaking. It is characterized by large velocities (magnitude and direction); $\mathbf{u}^{w}$ is the irrotational or wave component of the velocity. The latter is smaller than $\mathbf{q}^{D}$. The smallest component is $\mathbf{q}^{C}$, which represents the rotational component (possibly including an

(a)

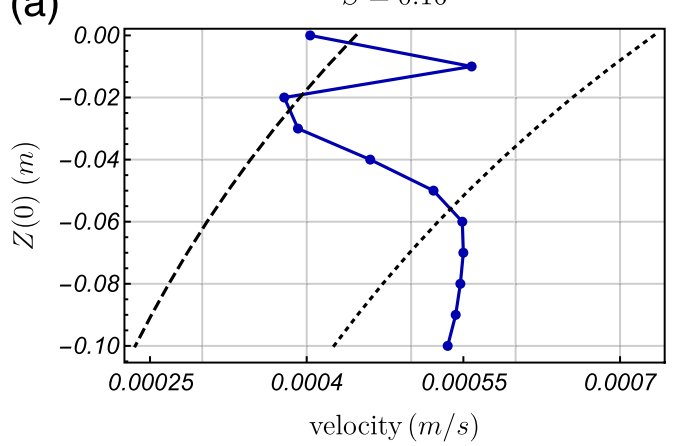

imposed current $\mathbf{v}^{C}$ ) as well as the velocity associated with transport due to waves and the residual flow due to breaking waves. [See also McWilliams and Restrepo (1999) and Restrepo et al. (2011): in these $\varepsilon \ll 1$ is the ratio of the current speed to the orbital wave speed.] At scales larger than wave scales, the $\mathbf{q}^{D}$ is unresolved and could be parameterized by a jump/diffusive stochastic process. In fact, we will adopt a zero-mean Wiener process to model this velocity (clearly, an ad hoc representation that can elicit a number of objections, the least of which is that it is incompressible only in the mean). Associated with the above velocity decomposition, the parcel path is represented as $\mathbf{Z}=\mathbf{Z}_{0}+\varepsilon \mathbf{Z}_{1}+\varepsilon^{2} \mathbf{Z}_{2}+\cdots$, and the time scales associated with these are the smaller, the wave scale, and the larger than wave scale, respectively.

We use the operator $\langle\cdot\rangle$ to denote ensemble average, which in this case means expectation with respect to the (b)

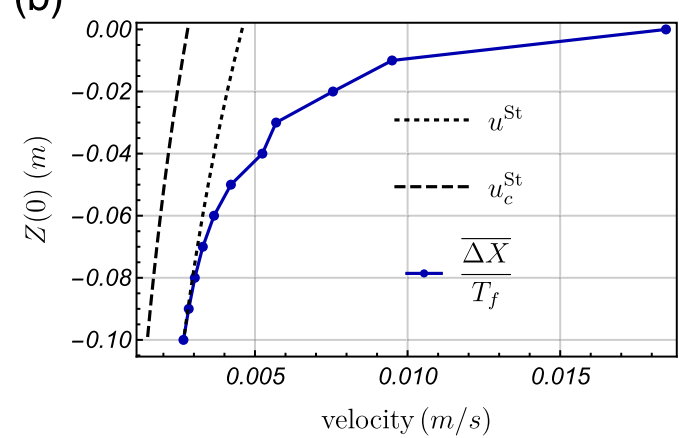

FIG. 2. Mean horizontal transport, from data, as a function of depth, compared with the Stokes drift (dashed curves) for the cases (a) $S=0.16$ and (b) $S=0.4$ (a breaking case). The heavier dashed curve corresponds to $\mathbf{u}_{c}^{\text {St }}$ computed using (6) with $\mathscr{D}=1$. The lighter dashed line corresponds to the Stokes drift $\left(1 / T_{f}\right) \int_{0}^{T_{f}} \int^{t} \mathbf{u}(x, z, s) d s \cdot \nabla \mathbf{u}(x, z, t) d t$, with $\mathbf{u}$ given by (1). Here, $T_{f}=35 \mathrm{~s}$ and the data mean here is taken over all paths with same initial depth. The Stokes drift velocity $\mathbf{u}_{c}^{\text {St }}$ is computed using (6) with $\mathscr{D}=1$. (Note horizontal scales). The small $S$ mean transport is coincidentally well estimated in magnitude, particularly close to the surface, by the Stokes drift. This is not true for the large $S$ case, breaking or otherwise. 
stochastic component of $\mathbf{q}$, a Wiener noise process $\sqrt{2 D_{0}} W(t)$ satisfying $\langle W(t)\rangle=0$, and $\left\langle W(t) W\left(t^{\prime}\right)\right\rangle=t \delta_{t, t^{\prime}}$ (delta-correlated) and affecting $\mathbf{q}$ at the fastest time scales; $D_{0}$ is the corresponding dispersion rate. Further, for some $f(t), \bar{f}$ denotes the average of $f$ over the period $T_{c}=1 / \nu_{c}$ (the subscript $c$ on $\nu_{c}$ denotes the central frequency).

The critical observation in the Longuet-Higgins (1953) analysis is that, in the case of a monochromatic progressive wave, say

$$
\begin{aligned}
\mathbf{u}^{w}(x, z, t)= & -\nabla \frac{a_{c} \omega_{c}}{k_{c}} \frac{\cosh \left[k_{c}(z+h)\right]}{\cosh \left(k_{c} h\right)} \\
& \times \cos \left[k_{c}\left(x-x_{b}\right)-\omega_{c} t\right],
\end{aligned}
$$

the average $\overline{\mathbf{u}^{w}}$ vanishes. Following Restrepo (2007), the lowest-order Lagrangian and Eulerian velocities also vanish on average. Hence

$$
\begin{aligned}
\left\langle\overline{d \mathbf{Z}_{0}}\right\rangle & =\left\langle\overline{\sqrt{2 D_{0}} d \mathbf{w}_{t}}\right\rangle=0, \\
\left\langle\overline{d \mathbf{Z}_{1}}\right\rangle & =\left\langle\overline{\mathbf{u}^{w}\left(\mathbf{Z}_{0}, t\right) d t}\right\rangle=0, \quad \text { and } \\
\left\langle\overline{d \mathbf{Z}_{2}}\right\rangle & =\mathbf{v}^{C} d t+\mathbf{u}^{\mathrm{St}} d t,
\end{aligned}
$$

where $d \mathbf{w}_{t}:=\left(d W_{t}, 0\right)$, the subscript $t$ reminding us that this is an incremental Wiener process in time (in units of $\mathrm{m}^{1 / 2}$ ). Equation (5) has the imposed current (if present) and a residual flow due to wave breaking, as well as the Stokes drift velocity $\mathbf{u}^{\mathrm{St}}$. The Stokes drift $\mathbf{u}^{\mathrm{St}}=\left(\mathbf{u}_{c}^{\mathrm{St}}, 0\right)$ can be obtained by computing

$$
\mathbf{u}_{c}^{\mathrm{St}}=\left\langle\overline{\int_{0}^{t} \mathbf{u}^{w}\left(\mathbf{Z}_{0}, s\right) d s \cdot \nabla \mathbf{u}^{w}\left(\mathbf{Z}_{0}, t\right)}\right\rangle,
$$

where $\mathbf{Z}_{0}=\mathbf{Z}_{0}(0)+\sqrt{2 D_{0}} \mathbf{w}_{t}$. For progressive monochromatic waves, the steady Stokes drift velocity is

$$
\mathbf{u}_{c}^{\mathrm{St}}=\frac{S_{c}^{2} c_{p}}{2} \mathscr{D} \cosh ^{2}\left[k_{c}(z+h)\right] \operatorname{csch}\left(2 k_{c} h\right),
$$

with $S_{c}=k_{c} a_{c}, c_{p}=\omega_{c} / k_{c}$ is the phase speed, and $\mathscr{D}=1 /(1+\Delta)$, where $\Delta=k_{c}^{4} D_{0}^{2} / \omega_{c}^{2}$. We made use of $\omega_{c}=\sqrt{g k_{c} \tanh \left(k_{c} h\right)}$ in obtaining (6). The Stokes drift velocity is proportional to $a_{c}^{2}$ via $S_{c}^{2}$. Note that the stochastic term in the parcel path velocity leads to an increase in the variance of the Stokes drift. It also leads to a suppression of wave-generated transport (see Restrepo (2007)). The term $\mathscr{D}$ equals 1 when the stochastic process is zero, leading to the familiar expression for the Stokes drift velocity under progressive monochromatic waves. Condition (4) holds exactly for monochromatic waves and holds as well for random-phase, stationary linear waves (see Huang 1971; Kenyon 1969).

\section{Transport due to transient waves}

In the analysis of the data, the mean horizontal displacement is defined as $\left\langle X\left(T_{f}\right)-X(0)\right\rangle$. We also define transport as the mean horizontal velocity $\left\langle X\left(T_{f}\right)-X(0)\right\rangle / T_{f}$ over the whole simulation. In this context, the ensemble average $\langle\cdot\rangle$ of a quantity is estimated as the average of such quantity over all paths with initial location within the central light shaded rectangle of black points in Fig. 1. The assumption here is that the uncertainty modeled as a stochastic component in $\mathbf{q}$, translates into the data as uncertainty over the parcel path's initial condition $\mathbf{Z}(0)$.

In what follows we describe essential aspects of the kinematics of the transport. We will use dimensional quantities. The average transport is obtained by averaging the computed parcel paths in (2). For transient waves, meaning waves for which (4) does not hold over the interval of time of interest, (3)-(5) do not apply and the Stokes drift, as defined above, makes no sense.

We found that by setting $\mathbf{q} \approx \mathbf{u}^{w}$, as given in (1), into (2) we were able to construct parcel paths that were qualitatively very similar to the data, regardless of which initial parcel path position we chose. The paths so constructed mimic the details in space and in time, for a large range of $S$, excluding breaking cases. The conclusion to be drawn from this is that, if dissipation and dispersion are appropriately parameterized, a linearized velocity model can capture most of the details of the parcel paths, in spite of the fact that the numerical solutions are those approximating the Navier-Stokes equations.

The fact that $\mathbf{q} \approx \mathbf{u}^{w}$ approximates the Lagrangian velocity implies that the horizontal displacement $d X$ is proportional to $S d t$, regardless of the value of $S$. As DPM17 found, when $S$ is small, we found that the mean transport is proportional to $S^{2}$, and when $S$ is large, the mean transport can be significantly larger, proportional to $S$. This is explained as follows: when $S$ is small, (4) is approximately satisfied. For example, near to the sea surface and for $S=0.16, \overline{U(t)} \approx 10^{-6}$ $\mathrm{m} \mathrm{s}^{-1}$. Hence (3)-(5) approximately hold and the Stokes drift is an approximation of the mean transport which is thus approximately proportional to $S^{2}$ as per (6). However, for $S=0.32, \overline{U(t)} \approx 10^{-3} \mathrm{~m} \mathrm{~s}^{-1}$. In this case the mean transport is proportional to the amplitude of $\mathbf{u}^{w}$ (i.e., to $S$ ) and is given by the average of $\dot{\mathbf{Z}}(t)$ in (2). This $S$ dependency holds for mean transport at any depth. 

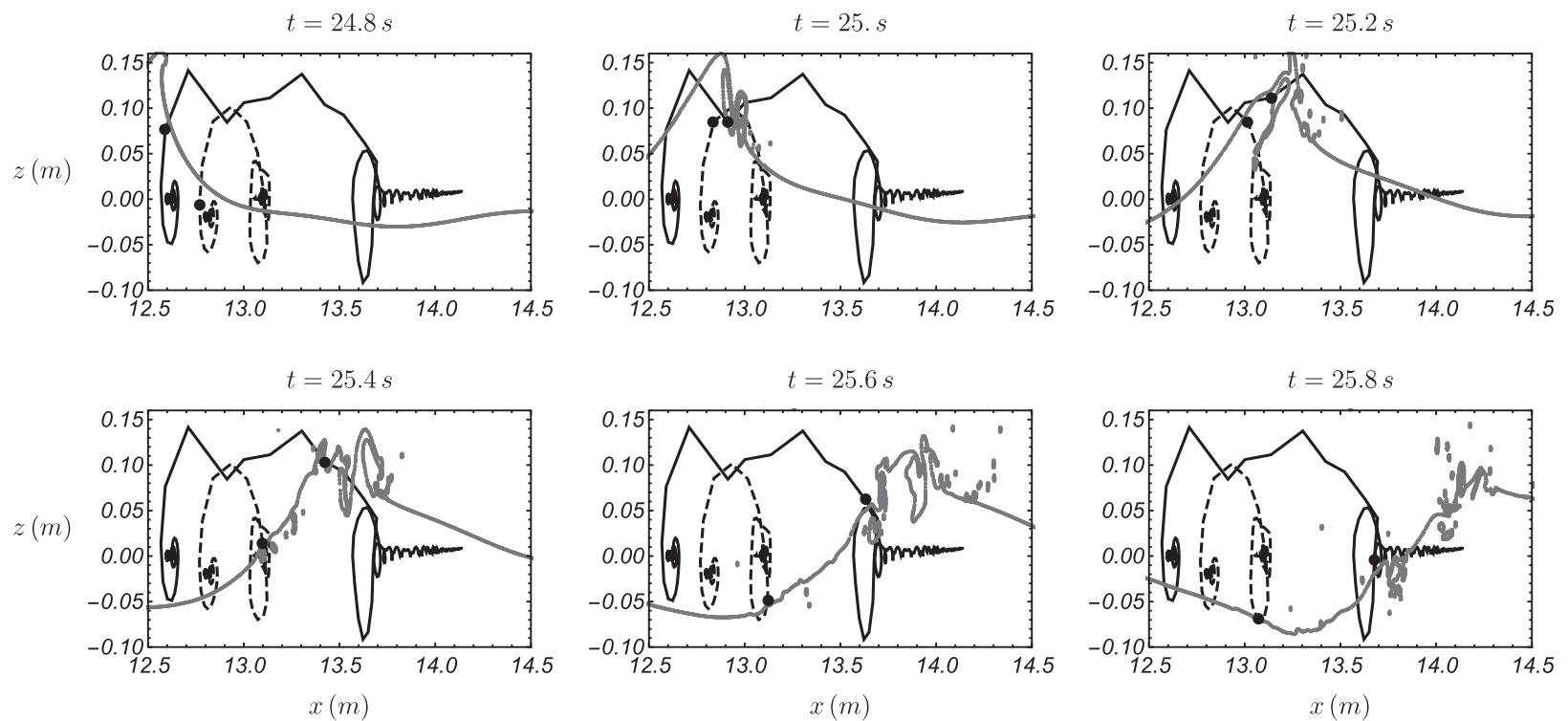

FIG. 3. Lagrangian parcel path histories for $S=0.4$, The breaking free surface is shown in gray. The smoother path (dashed line) that follows an elliptical path punctuated by a large displacement is most typical of parcel paths, throughout. The more complex (solid line) path is of the type we denote as a rider parcel path, since it spends some time on the sea surface. It started near $x_{b}$ on the sea surface.

Figure 2 depicts the mean transport as a function of depth and an estimate of the same using the Stokes drift velocity, for two values of $S$. In each of the panels, the solid line corresponds to the mean transport estimated using the parcel path data, and the dashed curves two different estimates of the transport using the Stokes drift. The heavy dashed line corresponds to $\mathbf{u}_{c}^{\text {St }}$, computed using (6) with $\mathscr{D}=1$. The lighter dashed line is the estimate of the mean transport obtained by computing $\left(1 / T_{f}\right) \int_{0}^{T_{f}} \int^{t} \mathbf{u}(x, z, s) d s \cdot \nabla \mathbf{u}(x, z, t) d t$, with $\mathbf{u}$ given by (1). Note that for large $S$ the transport can be 3 times larger in the near-surface region when compared with deeper paths. For small $S, \overline{U(t)} \approx 0$, which is consistent with the parcel paths being very similar to the familiarlooking paths of monochromatic waves, hence for small $S$ the Stokes drift velocity is quantitatively similar to the mean transport. As we will explain later, for large $S$ the basic assumptions for the validity of the Stokes drift velocity to be an estimate of the mean transport are not satisfied.

Figure 3 shows a couple of parcel paths for large $S$, that is, highly transient waves (see also Figs. 4 and 6). The gray line demarcates the (breaking) free surface. A dot indicates the parcel position at the time specified by the frame. The solid path corresponds to a wave-riding parcel. These parcels spend some time on the free surface, right at the moment when the breaking occurs, resulting in a complex displacement history (see Pizzo 2017). The parcel path marked as a dashed line is taken from an initial condition with $Z(0)=-0.04$. The large excursion coincides with the passage of the wave. The dashed path is more typical of the parcel path dynamics inside the water column for large $S$ including breaking waves. Figure 4 features several parcel paths for the $S=0.4$ (breaking) case in different regions within the tank. The first row corresponds to parcel paths starting at the surface. Paths in the two lower rows correspond to parcel paths originating below the near-surface region in Fig. 1 and are very typical of the paths under large unbreaking as well as breaking waves. The dashed portion highlights the parcel flight that coincides with the wave passing overhead. Clearly, it is this largeamplitude displacement and the residual motion after the wave passage that largely accounts for significant mean transport.

For very small $S$, as a function of depth $z$ the mean transport has the familiar $\exp \left(2 k_{c} z\right)$ dependence, independent of $x$. For large $S$ the vertical profile has an exponential profile, but the rate of change in $z$ and $x$ is more complex. Figure 5 highlights the depth dependence of the total displacement at different locations $x$ along the tank. The parcel data used in the estimated displacement were located at $t=0$ at $X(0)=10.6,12.4$, and $14.2 \mathrm{~m}$, respectively. Dividing by $T_{f}$ yields an estimate of the mean transport.

If $S>S_{0}$, wave breaking can occur and vorticity is generated in the flow but found confined to a very thin layer close to the sea surface. This vorticity lingers after the wave passes. Wave breaking enhances dispersion, regardless of the manner in which the waves break, but 

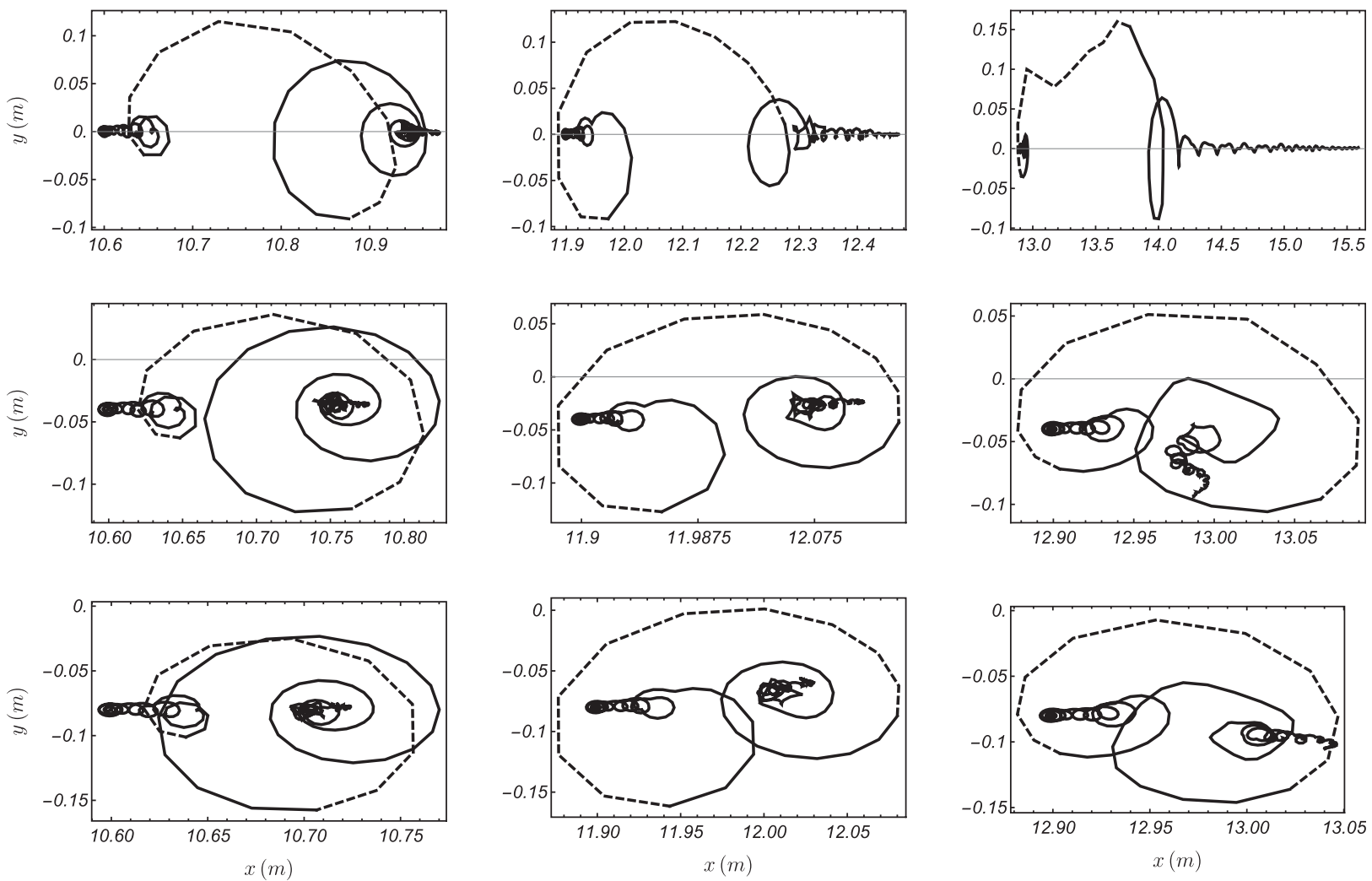

FIG. 4. Lagrangian parcel path histories corresponding to different initial locations. The wave slope is $S=0.4$. Highlighted as dashed is the portion of the path when the crest of the wave passes overhead.

it is the ensuing vorticity that enhances transport: wave momentum is transferred to the mean flow (see Fig. 6).

A time series of the horizontal component of the velocity, following one of the parcels is shown in the left panel of Fig. 7. The right panel of Fig. 7 depicts the horizontal velocity obtained from solving

$$
\dot{\mathbf{Z}}=\mathbf{u}^{w}[\mathbf{Z}(t)],
$$

with $\mathbf{u}^{w}$ given by the linearized wave field in (1), and $Z(0)=-0.07 \mathrm{~m}$. The two are qualitatively similar, however, the time series associated with (7) severely overestimates the data velocity for large $S$. Evidently, surface breaking, and the generation of vorticity/dissipation inherent in the Navier-Stokes solution has a significant effect on the amplitude of the parcel path. Equation (7) forms the basis for a transient transport model to be introduced in a subsequent Section. A simple parametric model for dissipation is proposed to improve the compatibility of (7) with regard to predicting mean transport at any depth for any $S$. We propose a modification to (1) that includes dissipation parametrically as follows
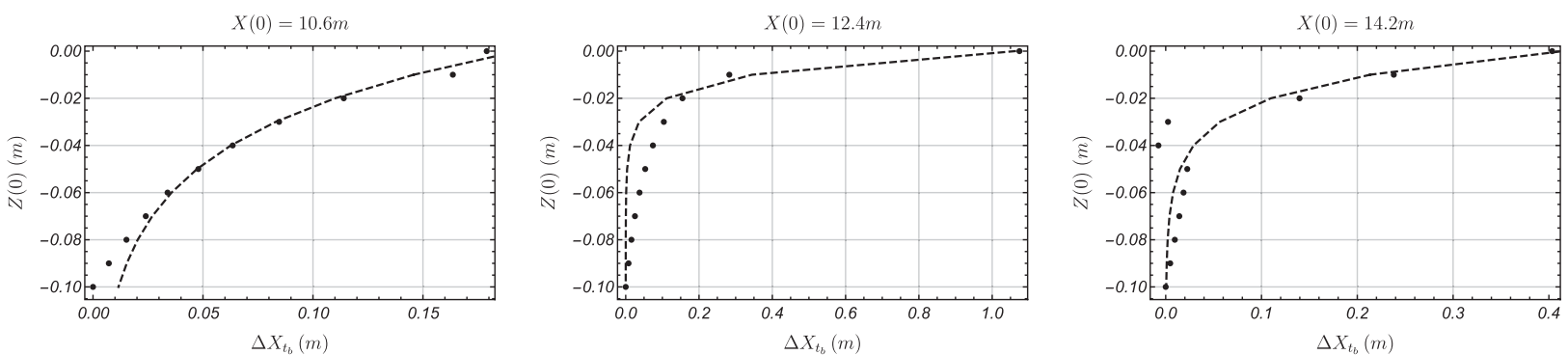

FIG. 5. Total displacement $\Delta X_{t_{b}}$ in the $x$ direction during the period of the path when the crest goes over the particle (dashed curves in Fig. 4), as a function of depth, for the case $S=0.4$ and three different values of initial $X(0)$. The averaged data appears as points, and the fitted exponential as a solid curve. The exponential fits are $0.053+0.1932 \exp (28.153 z), 0.111+1.062 \exp (112.626 z)$, and $0.0911+0.415 \exp (66.514 z)$ for $X(0)=10.6,12.4$, and $14.2 \mathrm{~m}$, respectively. 

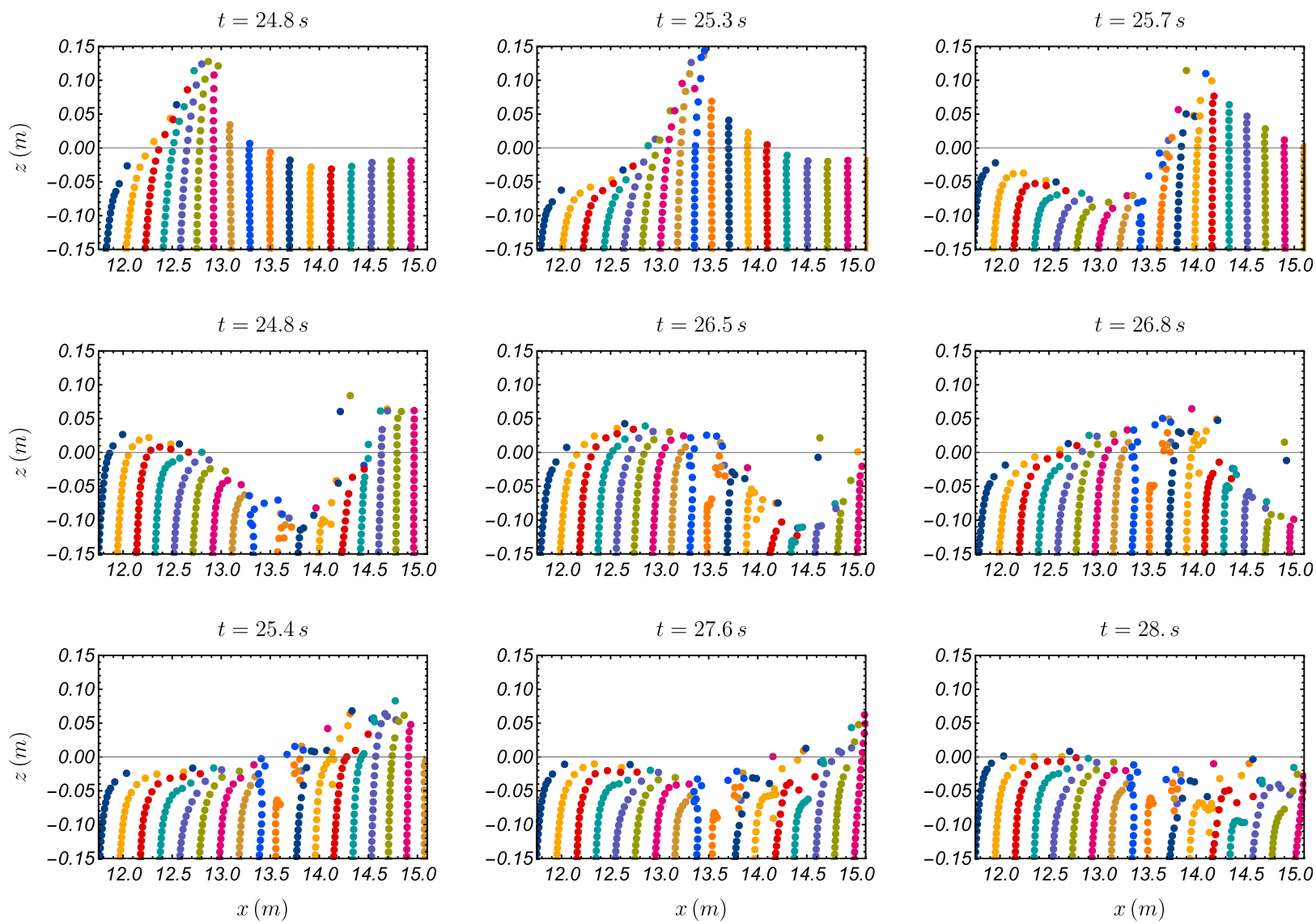

FIG. 6. Evolution of parcel paths for $S=0.4$. Transport and dispersion is evidenced by irreversible forward displacement of the parcels. Complex-motion displacements are found to be confined to a very thin layer close to the free surface.

$$
\begin{aligned}
\dot{\mathbf{Z}}= & \mathbf{u}_{d}^{w}[\mathbf{Z}(t)]=\nabla \phi_{d}[\mathbf{Z}(t), t], \\
\phi_{d}= & -\sum_{n=1}^{N} \frac{a_{n} \omega_{n}}{k_{n}} \frac{\cosh \left[k_{n}(z+h)\right]}{\sinh \left(k_{n} h\right)} \\
& \times \sin \left[k_{n}\left(x-x_{b}\right)-\omega_{n} t\right] e^{-\beta k_{n}^{2} t},
\end{aligned}
$$

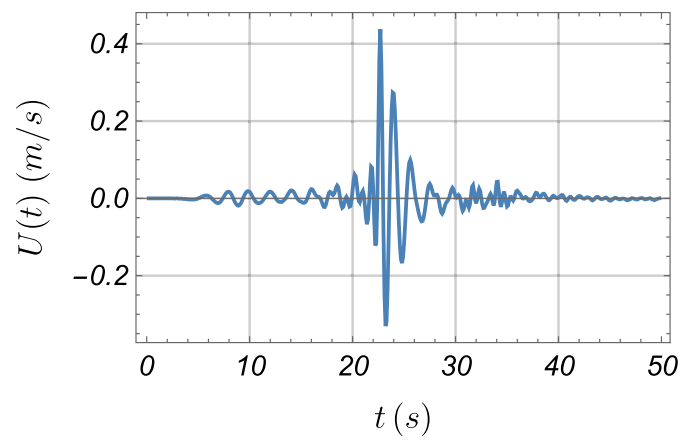

where $\beta$ is a tunable dissipation parameter. In section 5 we provide further details on how this parameter is chosen. The essential lesson is that even though the numerical code is solving Navier-Stokes, for smalland large-amplitude waves, a simple model for the Lagrangian velocity captures the paths qualitatively

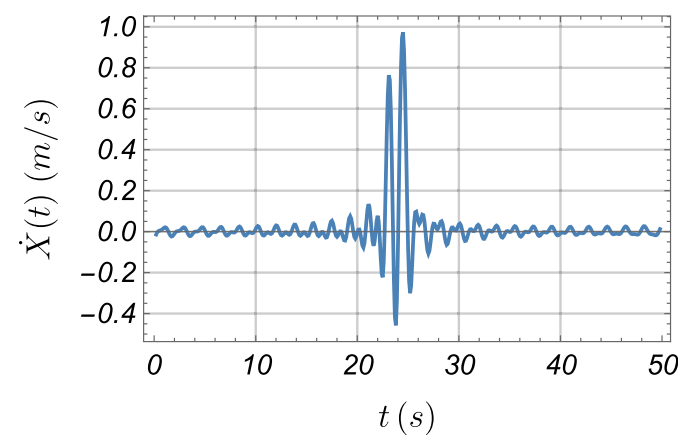

FIG. 7. (left) Horizontal Lagrangian velocity (data) of a parcel with $\mathbf{Z}(0)=(10.6,-0.07) \mathrm{m}$ in the case $S=0.4$. (right) Horizontal Lagrangian velocity, obtained by numerically solving $\dot{\mathbf{Z}}=\mathbf{u}^{w}[\mathbf{Z}(t)]$ with $\mathbf{u}^{w}$ given by (1). Note the difference in vertical scale. The spectrum of the forcing boundary condition creates a transient velocity everywhere in the tank. 

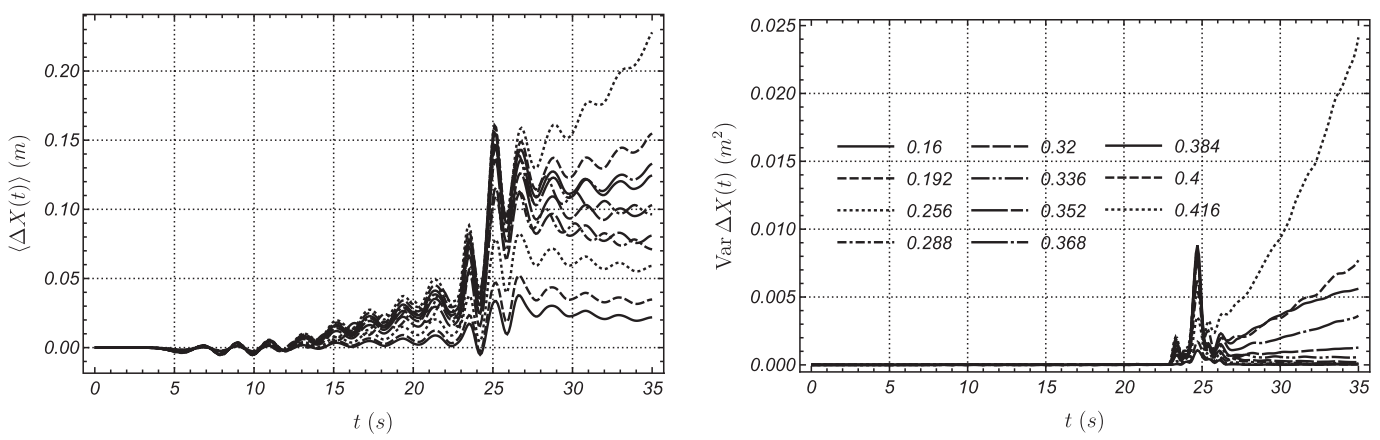

FIG. 8. (left) Mean and (right) variance of the horizontal displacement $\Delta X(t)=X(t)-X(0)$ as a function of $S$. Averages are taken over the trajectories starting in points within the gray-shaded middle area of Fig. 1. The breaking cases correspond to $S>S_{0}=0.336$. The dispersion persists after the wave breaks, for breaking cases.

mostly everywhere in the tank at any time and can be tuned to deliver reasonable mean transport estimates for a large range of $S$. How close the model captures the mean transport will be touched upon in section 5 , when inclusion of wave-breaking dispersion is taken into account.

Figure 8 shows the time dependent mean and variance of the (horizontal) displacement, estimated from data. Large horizontal displacements are caused by largeamplitude and highly transient waves. On the right we present the horizontal displacement variance. Before the wave passes overhead, the variance is nearly zero. Once the wave passes overhead, there is an injection of variance. For nonbreaking cases, the variance reverts back to nearly zero, after the wave passes. However, this is not the case for breaking cases: the variance grows linearly, in fact. Wave breaking generates variability in the horizontal transport, coinciding with increased vorticity.

\section{The transport model}

The goal in this section is to extend the model (8) and (9) to increase the range of $S$ for which the model can capture the mean transport due to transient progressive waves, to include breaking waves. The model is tuned to deliver the correct mean transport but will be based on good qualitative approximations of the parcel paths, for all values of $S$. That is, a good approximation of the mean transport for any $S$, regardless of whether $\overline{\mathbf{Z}_{1}}$ is small or otherwise. A model, consistent with the observations made in section 4 , is

$$
\langle d \mathbf{Z}\rangle=\mathbf{v}^{C} d t+\left\langle\mathbf{u}_{d}^{w}(\mathbf{Z}, t) d t+\sqrt{2 D\left(t-t_{b}\right)} d \mathbf{w}_{t}\right\rangle .
$$

The first term on the right hand side is zero in the data, but otherwise, would represent an imposed current. The model has two fitting parameters: the dissipation rate $\beta$ and the molecular diffusion $D(t)=D_{b} \Theta\left(t-t_{b}\right) \Theta\left(S-S_{0}\right)$, where
$\Theta$ is the Heaviside function, $S_{0}$ is the threshold $S$ for wave breaking, and $D_{b}>0$. The physical units of the variance strength $D$ are meters squared per second squared $\left(\mathrm{m}^{2} \mathrm{~s}^{-2}\right)$. The parameters $\beta$ and $D_{b}$ were estimated from data: we used the data to estimate the ensemble average $\left\langle X\left(T_{f}\right)-X(0)\right\rangle$ and then solved $d \tilde{\mathbf{Z}}=$ $\mathbf{u}_{d}^{w}(\tilde{\mathbf{Z}}, t) d t$ for the same initial locations, and numerically estimated the value of $\beta$ such that $\left\langle\tilde{X}\left(T_{f}\right)-\tilde{X}(0)\right\rangle$ gave the best approximation to the average from the data. Figure 9 compares the fit of $\beta$, as a function of $S$, when the model is tuned to data that includes nearsurface paths (triangles) and when it does not (circles). The values are similar. The fit leads to $\beta \approx 0.0024$ $\mathrm{m}^{2} \mathrm{~s}^{-1}$. The value of $D_{b}(S)$ was estimated following the same fitting methodology using instead the second moment $\left\langle\left[X\left(T_{f}\right)-X(0)\right]^{2}\right\rangle$. A linear fit yields $D_{b}(S)=$ $0.0052 S-0.0018$, valid for $S>S_{0}$, where $S_{0}$ is the breaking threshold slope. It is practically zero for $S<S_{0}$. (The range of $D_{b}$, as a function of $S$, is so small that it might be approximated by a constant: $D_{b} \approx 2 \times 10^{-4} \mathrm{~m}^{2} \mathrm{~s}^{-2}$ ). Note that this particular numerical approximation depends on the units used for $D_{b}$, but the qualitative observation remains.

We will denote the model given by (10) as the subsurface mean transport model, in order to emphasize that this model tracks paths well for most $S$, including in the regime of $S$ leading to breaking waves, but does not apply to the near-surface (see Fig. 1). This is because it does not take into account transport due to parcels that initiate in near-surface zone. As will be shown, the model captures the mean transport and dispersion, by virtue of the tuning of the parameters, for any $S$, below the zone highlighted in gray in Fig. 1.

Figure 10 compares data to paths from a single realization of the subsurface model in two contrasting cases: a large-amplitude, nonbreaking case, $S=0.26$, in Fig. 10a, and a breaking case, $S=0.4$, in Fig. 10b. The data and the model outcome are similar qualitatively, 


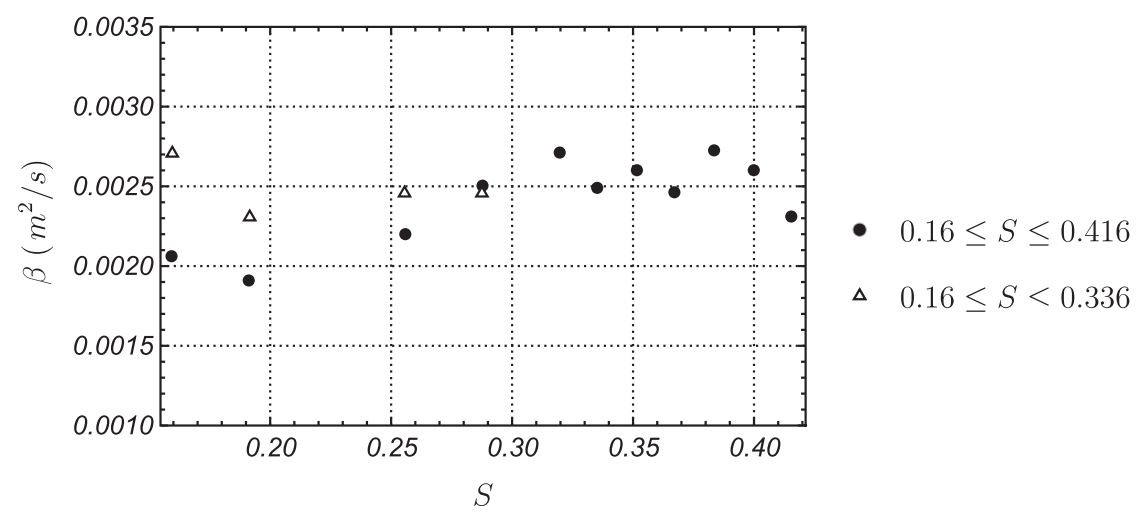

FIG. 9. Fitted $\beta$ values as a function of $S$. For nonbreaking cases (triangles) we included paths up to the surface in the estimate of $\beta$. Dots mark the estimates of $\beta$ for all values of $S$ and paths initially in the central gray-shaded area of Fig. 1.

and close quantitatively. Though not shown, for small $S$, the agreement between model and data is even better. Figure 11 compares ensemble displacement and variance of the data and model runs for $S=0.16,0.256$, 0.336 , and 0.416 , corresponding to two nonbreaking cases and two breaking cases, respectively. The ensembles encompass data and model outcomes for all parcels with starting points indicated by dots in Fig. 1, but lying below the gray zone.

For nonbreaking cases, $S<S_{0}=0.336$, the model is able to predict the statistics of transport throughout the water column, including in the near-surface region. Figure 12 displays comparisons of data and subsurface model outcomes of the mean horizontal displacement and its variance, as a function of the slope $S$. In the top panels the experimental mean transport and variance is computed using all of the parcels, including parcels whose initial starting location was on the surface or in the area shaded in gray in Fig. 1. The model compares favorably with the data. The model predicts that the transport is proportional to $S$, for any $S$ below $S_{0}$, regardless of whether it is large or small, and that the mean transport is proportional to $S^{2}$, for small $S$ and proportional to $S$, for large $S$. In the lower panels of Fig. 12 we compare the mean transport and variance of the data and the model for the full range of $S$. In these comparisons we exclude data and model outcomes from the near surface (gray region).

\section{Discussion and summary}

We analyzed numerical simulations of the mean transport due to a transient, progressive wave packet. The flow was produced by a boundary-driven Navier-Stokes solver capable of resolving the free surface dynamics. In the simulations the Reynolds number was approximately 90000 . The Reynolds number was moderately high but it must be emphasized, it is not the issue that limits the applicability of the outcomes and analyses presented here, to laboratory or oceanic situations. Rather, it is that the simulated flow does not represent 3D turbulence generated by breaking wave accurately, as by virtue of
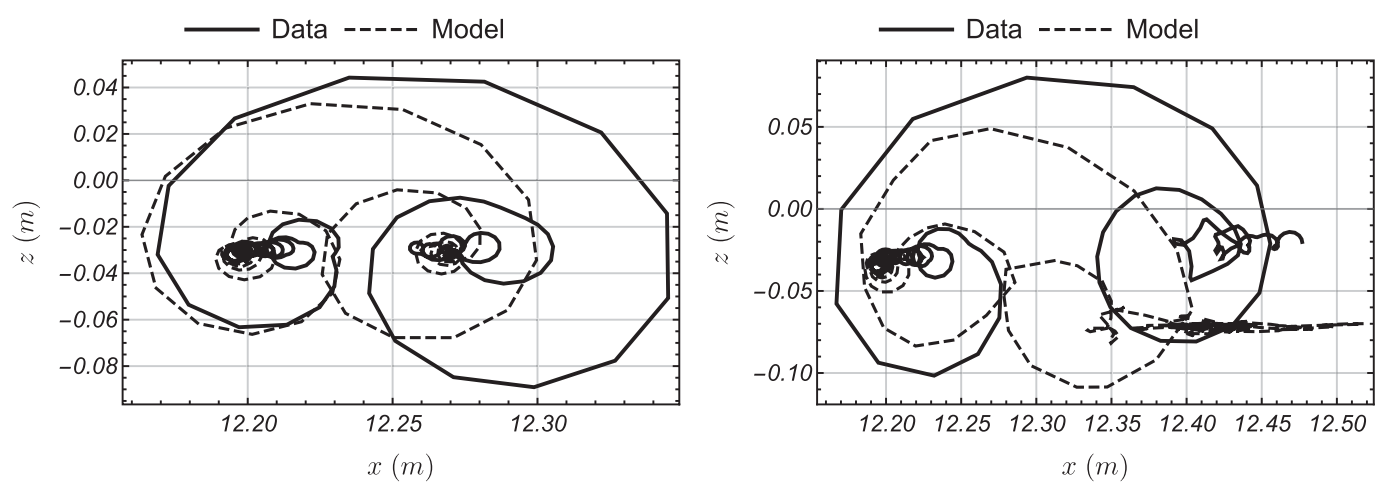

FIG. 10. Comparison of data [(2)] and the subsurface model (10) parcel paths, for (left) $S=0.26$ and (right) $S=0.4$; $\mathbf{Z}(0)=(12.2,-0.3) \mathrm{m}$ in both cases. 

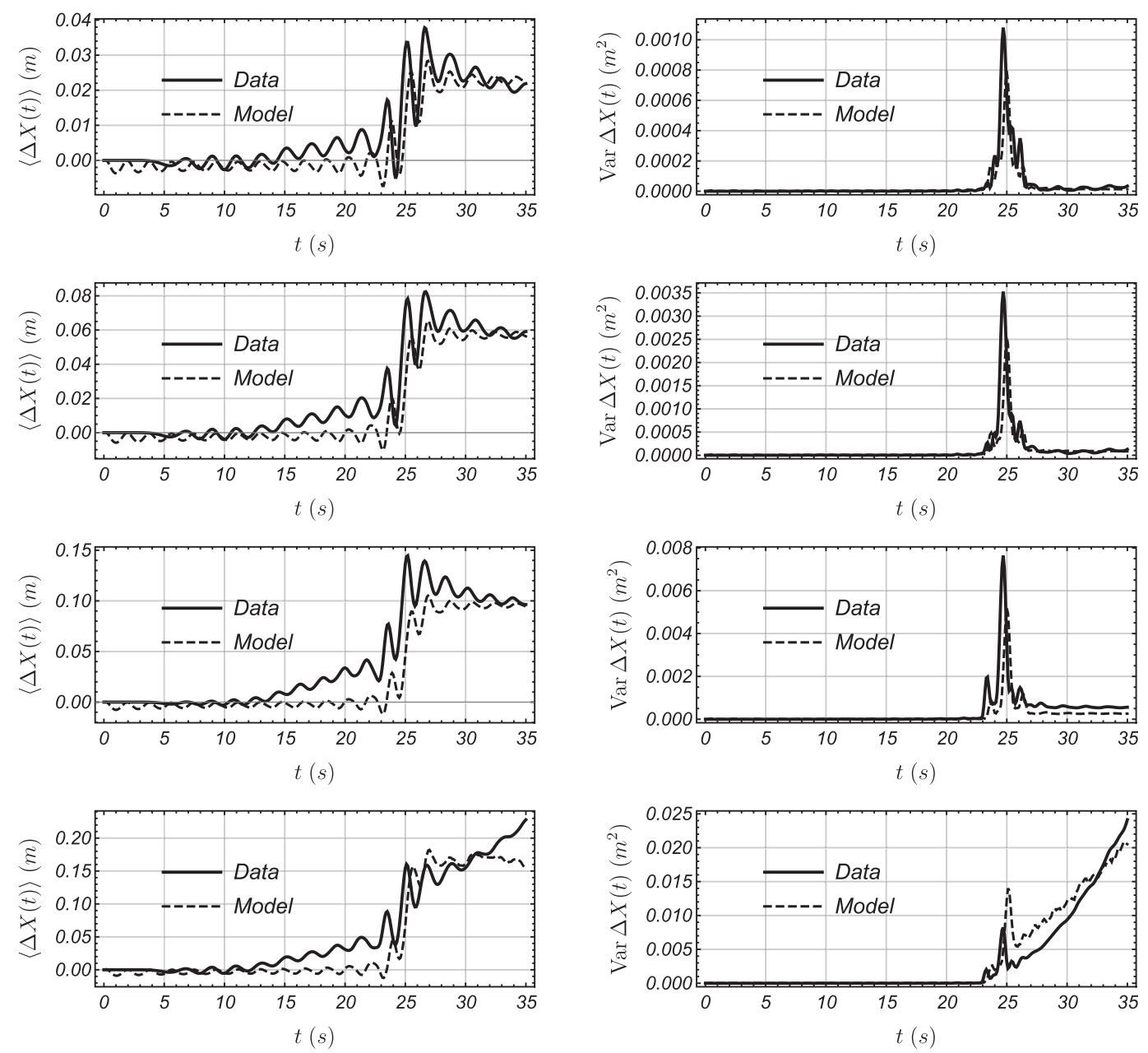

FIG. 11. (left) Ensemble mean of the horizontal displacement $\Delta X(t)=X\left(T_{f}\right)-X(0)$ and (right) its variance as a function of time. From top to bottom, $S=0.16,0.256,0.336$, and 0.416 , corresponding to two nonbreaking cases and two breaking cases. Averages are taken over all trajectories starting at points marked in Fig. 1.

being constrained to two space dimensions, it does not support vortex stretching.

The transport was analyzed by examining ensembles of the parcel paths. Parcel paths were computed numerically using the velocity field obtained by the Navier-Stokes solver. The simulations (data) we analyzed here were examined in and provided by DPM17.

In their simulations the velocity boundary forcing had a parameter $S$, they called the wave slope, which controlled the wave amplitude of the progressive waves it generated. The parameter $S$ also affected the relative strength of the spectral components of the multichromatic forcing and thus the transient characteristics of the propagating wave. For $S$ small the boundary forcing produced wave packets that were nearly indistinguishable from steady progressive waves. As $S$ increased, the transient wave packet nature of the waves became apparent. The wave packets peaked at a prescribed location and time in the numerical wave tank. When $S$ was very large the boundary forcing would produce waves with the propensity to break.

A key conclusion in DPM17 was that mean transport was found to exhibit a sharp transition: proportional to $S$ for breaking waves and to $S^{2}$ when waves did not break (see their Fig. 8). The change between these two was abrupt and coincided with a critical value of $S=S_{0}$. The DPM17 conclusions were guided by approximate dimensional arguments, for breaking waves, which happen to correspond to large $S$ cases, along with the application of an analysis of parcel paths in Pizzo (2017) [see Eq. (224) of Pizzo (2017) and Eq. (4.1) of DPM17] of the John equation for surface Lagrangian velocities (see John 1953). The transport is proportional to $S$ in the John equation, regardless of the magnitude of $S$. The equation, however, does not suggest without further work, how 
(a)

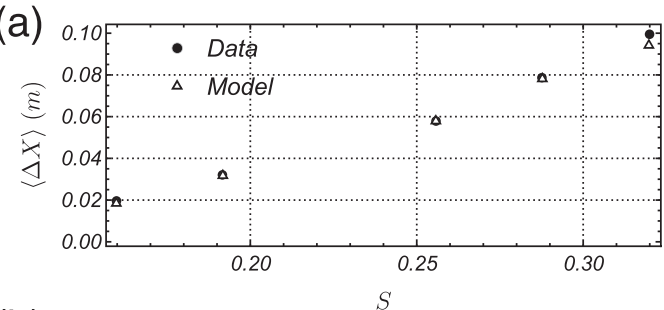

(b)

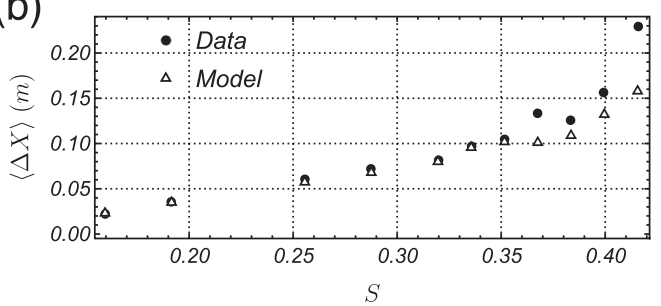

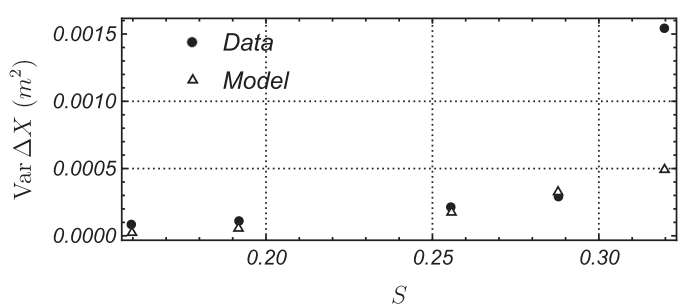

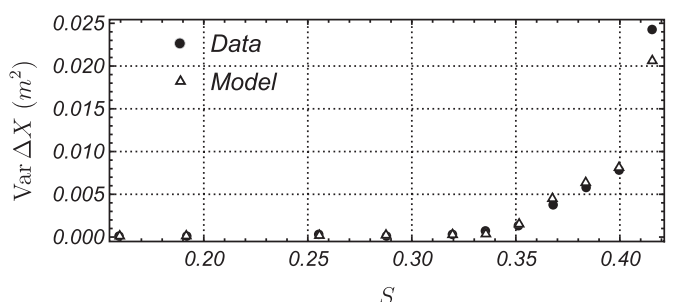

FIG. 12. Comparisons of the depth-averaged (left) mean horizontal displacement $\Delta X(t)=X\left(T_{f}\right)-X(0)$ and (right) variance, as a function of slope $S$. Averages are taken over the trajectories starting in positions marked as black in Fig. 1. (a) A comparison of the mean and variance of the data vs the model, up to but not including the range of $S$ that includes wave breaking. (b) The mean and variance of the data and the model are compared for the full range of $S$, but exclude data/model data from parcels that started near the surface (in the gray-shaded region of Fig. 1.

the mean transport is proportional to $S$, for large $S$, and to $S^{2}$ for small $S$.

Our analysis of their data finds that indeed, for small $S$ the mean transport is approximately proportional to $S^{2}$. However, this is because for small $S$ the waves were approximately monochromatic progressive waves of steady form. As $S$ increases, the mean transport becomes proportional to $S$ even when no breaking occurs, and further, the transition from $S^{2}$ to $S$ transport transitions smoothly. We explain this by noting that for monochromatic progressive waves the first-order transport average is zero and thus the leading-order mean transport is the Stokes drift. In contrast, for the transient waves the leading-order mean transport is not zero and this leading-order mean transport is proportional to $S$. When $S$ was large enough that the waves broke, localized rotational effects were evident in the Navier-Stokes simulations and droplet ejection would sometimes occur. In these cases our analyses is not complete or applies very tentatively (i.e., the transport is proportional to $S$, up to the breaking time, and the mean transport is not well estimated because the breaking events sometimes lead to a rotationally induced residual flow that was seen to occur after the wave packet peak had passed). In the ensemble sense we can measure that the most dramatic difference between the mean transport due to nonbreaking and breaking waves, occurs in the variance of transport (see Fig. 8). It changes from being simply episodic in the nonbreaking cases, to persistent dispersion for high values of $S$.

We also showed that, regardless of whether waves were breaking or not, the parcel path evolution could be approximated, at least qualitatively, by replacing $\mathbf{q}(\mathbf{Z}, t) \approx \mathbf{u}^{w}(\mathbf{Z}, t)$ in (2), where $\mathbf{q}$ is the Eulerian velocity as approximated by the Navier-Stokes solver, and $\mathbf{u}^{w}$ describes the velocity of infinitesimal progressive waves (the boundary forcing is generated by $\mathbf{u}^{w}$ ). This approximation held for every parcel in the numerical wave tank, and for all values of $S$, so long as there was no free surface breaking. Using the velocity approximation, for $S<S_{0}$ we are able to replicate qualitatively the behavior of the mean transport with $S$. For small $S$ and for parcels far from the sea surface the above approximation is even quantitatively close. However, for $S>S_{0}$, in the range of $S$ in which wave breaking occurs, the approximation still held but not for parcels in the near-surface layer.

For large $S$ and for parcels starting at or very near to the free surface, the estimate $\dot{\mathbf{Z}} \approx \mathbf{u}^{w}(\mathbf{Z}, t)$ overestimates the extent of the orbital paths, and does not capture the resulting dispersion of the ensemble of paths. Apparently boundary layer effects and the generation of vorticity (not shown in this work but confirmed in the simulations) are but two manifestations of the difference between the Navier-Stokes velocity $\mathbf{q}$ there and the approximation $\mathbf{u}^{w}$. The parcel paths, near the free surface, for $S>S_{0}$ cases, can be tortuous, exhibit large variance, and thus cannot produce orbits as large as those given by $\dot{\mathbf{Z}} \approx \mathbf{u}^{w}$. This observation is consistent with established notions on wavebreaking phenomena that describe aspects of the wave energy and momentum transfer to the mean flow, and the resulting generation of shearing and vorticity (see Craig and Banner 1994; see also the review in Melville 1996).

Better quantitative comparisons of the mean transport were obtained when we incorporated an empirical 
dissipation term into the velocity: we replaced $\mathbf{u}^{w}(\mathbf{Z}, t)$ by $\mathbf{u}_{d}^{w}(\mathbf{Z}, t)$. See (9). The empirical dissipation term takes every $n$ component in the spectrum of the linearized irrotational velocity and multiplies it by $\exp \left(-\beta k_{n}^{2} t\right), \beta>0$ a fitted constant. This is, arguably, a simplistic model for dissipation for Navier-Stokes solutions, especially those involving wave breaking, however, it is telling that standard linear damping is all that is necessary to get good mean transport results and that this damping is very mild. The addition of the damping term, however, does not capture the dispersion evidenced in the data for large $S$ cases (particularly if the waves break). When $S$ is large vorticity appears very close to free surface, and intermittent dispersion is evidenced in the data. When $S>S_{0}$, vorticity and dispersion appear and linger, even after the passage of the breaking wave. Hence, the breaking waves introduces nontrivial parcel dispersion. There is an exchange of momentum between waves and the mean flow and the $\mathbf{q}(\mathbf{Z}, t) \approx \mathbf{u}_{d}^{w}(\mathbf{Z}, t)$ remains qualitatively acceptable, so long as it is not used to capture the paths of parcels that begin close to the free surface or on the free surface. Lagrangian paths computed by integrating the undampened $\mathbf{u}^{w}(\mathbf{Z}, t)$ show much higher transport than that observed in the data, and further, this approximation does not take into account dispersion.

These observations form the basis for a simple parametric stochastic mean transport model, applicable for any $S$ (although crude for the breaking wave case). Our model compares favorably with the data and the model is based upon our analysis of the kinematics. The model clearly produces mean transport proportional to $S$ for large $S<S_{0}$, breaking is not necessary to produce mean transport proportional to $S$, as is suggested in DPM17. The parcel path velocity is modeled by a combination of the damped velocity $\mathbf{u}_{d}^{w}(\mathbf{Z}, t)$ and a stochastic term that generates parcel dispersion and introduces vorticity in the resulting Eulerian velocity. The ensemble average of the model leads to a simple model of the mean transport that agrees with the mean transport in the data. Specifically, the model [see (10)] compares favorably with data in estimating the mean transport for small $S$ and for large $S$, even when breaking occurs (see Figs. 11, 12). The model yields good estimates of mean transport at any depth, including at the surface, so long as breaking is excluded. However, it also yields good results even when breaking takes place, so long as the transport is computed outside of the wave layer. The stochastic aspect of the model is crude but suggests that a stochastic modeling approach might be useful in capturing parametrically the effect of wave breaking on transport and dispersion, albeit in the mean. Agreement between the data and the model was achieved by tuning two model parameters. The parameters in the model were easy to tune to the data, however, it is not clear how well the model and its tuning would fare in an oceanic setting. Nevertheless, the utility of the model lies mostly in its ability to explain the details of the transport in the whole tank and for the whole range of $S$ (the whole tank for $S<S_{0}$ ).

In contrast to the steady, ideal nonbreaking translationally invariant or weakly invariant progressive wave case, for which the mean transport is approximated by the Stokes drift, regardless of wave amplitude, transient wave packets can produce more (localized) transport, and for large waves, more dispersion. This outcome obviously depends strongly on the transient nature of the wave itself. It was the case for the breaking and large nonbreaking wave cases considered here. While wave breaking is a very important type of transient wave phenomena and the ultimate current challenge in modeling its transport and its dispersion, in oceanic situations nonbreaking transient waves events are common, and thus understanding under what circumstances transients produce significant transport is worth further consideration. It is hoped that the present work helps in the analysis of transport due to transient waves and presents a conceptual roadmap for its representation through a model. Because the circumstances depend on the time spans in question, it is perhaps best to study transient transport as it relates to specific phenomena. For example, one might want to determine how Langmuir turbulence and oceanic processes that depend critically on wave-generated transport are affected by waves with transient behavior. The same sort of question could apply to the transport and dispersion of tracers, such as pollutants and of phytoplankton, and to air-sea exchanges. Perhaps the most obvious question generated by this work is how relevant are the results of this analysis to the oceanic situation. The key reason why the results of these experiments cannot be easily related to the oceanic situation is that these two-dimensional calculations, albeit derived from approximations of NavierStokes, do not support vortex stretching, and thus cannot represent turbulence appropriately, o support many of the complexities in the flow that are known to be critical to wave-breaking kinematics. Nevertheless, we believe that we largely succeeded in suggesting that there are discoveries to be made concerning transport in transient situations and these arise in a variety of geophysical fluid mechanics situations.

Acknowledgments. We thank L. Deike and K. Melville for sharing their data and for helpful discussions. We also thank James C. McWilliams for stimulating discussions and for suggesting improvements to the manuscript. An anonymous reviewer made many 
and significant comments and suggested changes that when addressed, improved the presentation as well as the breadth of its intended audience. This work was supported through a grant by the National Science Foundation, NSF OCE1434198. JMR wishes to thank the Kavli Institute of Theoretical Physics at the University of California, Santa Barbara. The KITP is supported in part by the National Science Foundation under Grant NSF PHY-1748958.

\section{REFERENCES}

Andrews, D. G., and M. E. McIntyre, 1978: An exact theory of nonlinear waves on a Lagrangian-mean flow. J. Fluid Mech., 89, 609-646, https://doi.org/10.1017/S0022112078002773.

Bühler, O., 2014: Waves and Mean Flows. Cambridge University Press, $370 \mathrm{pp}$.

Craig, P. D., and M. L. Banner, 1994: Modeling wave-enhanced turbulence in the ocean surface layer. J. Phys. Oceanogr., 24, 2546-2559, https://doi.org/10.1175/1520-0485(1994)024<2546: MWETIT>2.0.CO;2.

Deike, L., N. Pizzo, and W. K. Melville, 2017: Lagrangian transport by breaking surface waves. J. Fluid Mech., 829, 364-391, https://doi.org/10.1017/jfm.2017.548.

Huang, N. E., 1971: Derivation of stokes drift for a deep-water random gravity wave field. Deep-Sea Res. Oceanogr. Abstr., 18, 255-259, https://doi.org/10.1016/0011-7471(71) 90115-X.

John, F., 1953: Two-dimensional potential flows with a free boundary. Commun. Pure Appl. Math., 6, 497-503, https:// doi.org/10.1002/cpa.3160060405.

Kenyon, K., 1969: Stokes drift for random gravity waves. J. Geophys. Res., 74, 6991-6994, https://doi.org/10.1029/ JC074i028p06991.

Lane, E. M., J. M. Restrepo, and J. C. McWilliams, 2007: Wavecurrent interaction: A comparison of radiation-stress and vortex-force representations. J. Phys. Oceanogr., 37, 11221141, https://doi.org/10.1175/JPO3043.1.

Leibovich, S., 1983: The form and dynamics of Langmuir circulation. Annu. Rev. Fluid Mech., 15, 391-427, https://doi.org/ 10.1146/annurev.fl.15.010183.002135.

Longuet-Higgins, M. S., 1953: Mass transport under waves. Philos. Trans. Roy. Soc., 245A, 535-581, https://doi.org/10.1098/ rsta.1953.0006.

, 1970a: Longshore currents generated by obliquely incident sea waves: 1. J. Geophys. Res., 75, 6778-6789, https://doi.org/ 10.1029/JC075i033p06778. 1970b: Longshore currents generated by obliquely incident sea waves: 2. J. Geophys. Res., 75, 6790-6801, https://doi.org/ 10.1029/JC075i033p06790.

- , and R. W. Stewart, 1960: Changes in form of short gravity waves on long tidal waves and tidal currents. J. Fluid Mech., 8 , 565-583, https://doi.org/10.1017/S0022112060000803.

— and — 1962: Radiation stress and mass transport in gravity waves, with application to 'surf beats.' J. Fluid Mech., 13, 481-504, https://doi.org/10.1017/S0022112062000877.

_ , and - 1964: Radiation stresses in water waves: a physical discussion, with applications. Deep-Sea Res. Oceanogr. Abstr., 11, 529-562, https://doi.org/10.1016/0011-7471(64)90001-4.

McWilliams, J. C., and J. M. Restrepo, 1999: The wave-driven ocean circulation. J. Phys. Oceanogr., 29, 2523-2540, https://doi.org/ 10.1175/1520-0485(1999)029<2523:TWDOC > 2.0.CO;2.

_ P. P. Sullivan, and C.-H. Moeng, 1997: Langmuir turbulence in the ocean. J. Fluid Mech., 334, 1-30, https://doi.org/10.1017/ S0022112096004375.

_- J. M. Restrepo, and E. M. Lane, 2004: An asymptotic theory for the interaction of waves and currents in coastal waters. J. Fluid Mech., 511, 135-178, https://doi.org/10.1017/ S0022112004009358.

Melville, W. K., 1996: The role of surface-wave breaking in air-sea interaction. Annu. Rev. Fluid Mech., 28, 279-321, https:// doi.org/10.1146/annurev.fl.28.010196.001431.

Pizzo, N., 2017: Surfing surface gravity waves. J. Fluid Mech., 823, 316-328, https://doi.org/10.1017/jfm.2017.314.

Popinet, S., 2009: An accurate adaptative solver for surfacetension-driven interfacial flows. J. Comput. Phys., 228, 5838-5866, https://doi.org/10.1016/j.jcp.2009.04.042.

Restrepo, J. M., 2007: Wave breaking dissipation in the wavedriven ocean circulation. J. Phys. Oceanogr., 37, 1749-1763, https://doi.org/10.1175/JPO3099.1.

— J. M. Ramírez, J. C. McWilliams, and M. Banner, 2011: Multiscale momentum flux and diffusion due to whitecapping in wave-current interactions. J. Phys. Oceanogr., 41, 837-856, https://doi.org/10.1175/2010JPO4298.1.

Stokes, G. G., 1847: On the theory of oscillatory waves. Trans. Cambridge Philos. Soc., 8, 441-455.

Taylor, P. H., and T. S. van den Bremer, 2016: Lagrangian transport for two-dimensional deep-water surface gravity wave groups. Proc. Roy. Soc. London, 472A, 20160159, https://doi.org/ 10.1098/rspa.2016.0159.

Teixeira, M., and S. Belcher, 2010: On the structure of Langmuir turbulence. Ocean Modell., 31, 105-119, https://doi.org/ 10.1016/j.ocemod.2009.10.007.

van den Bremer, T. S., and O. Breivik, 2017: Stokes drift. Philos. Trans. Roy. Soc. London, 376, 20170104, https://doi.org/ 10.1098/rsta.2017.0104. 